\title{
ON BOUNDING HARMONIC FUNCTIONS BY LINEAR INTERPOLATION
}

BY H. F. WEINBERGER ${ }^{1}$

Communicated by E. Calabi, February 10, 1964

It is well known [1], [4] that Poisson's formula for the value at the origin $O$ of a function which is harmonic inside a circle $\left(x-x_{0}\right)^{2}$ $+\left(y-y_{0}\right)^{2}=A^{2}$ can be written in the form

$$
u(O)=\frac{1}{2 \pi} \int_{0}^{2 \pi} \frac{R(\theta+\pi) u(R(\theta), \theta)+R(\theta+\pi) u(R(\theta+\pi), \theta+\pi)}{R(\theta)+R(\theta+\pi)} d \theta,
$$

where $r=R(\theta)$ is the polar equation of the boundary. Thus the value of a harmonic function at any point in a circle is an average of the values obtained by linear interpolation of the boundary values at the ends of each chord through the point.

In particular, it follows that

$$
u(O) \leqq \max \frac{R(\theta+\pi) u(R(\theta), \theta)+R(\theta) u(R(\theta+\pi), \theta+\pi)}{R(\theta)+R(\theta+\pi)} .
$$

It is tempting to conjecture that a similar inequality holds for harmonic functions in any convex or even star-shaped domain. Recently J. Barta [2], [3] has given two incomplete proofs of this conjecture.

We shall show that in general no inequality of the form

(1) $u(O) \leqq M \max \frac{R(\theta+\pi) u(R(\theta), \theta)+R(\theta) u(R(\theta+\pi), \theta+\pi)}{R(\theta)+R(\theta+\pi)}$

can hold for all harmonic functions in a star-shaped domain $r<R(\theta)$. In fact, an inequality of the form (1) holds for each point $O$ of a convex domain $D$ only if $D$ is the interior of a circle.

We first prove:

Lemma. Let $G$ be the Green's function with singularity at $O$ for the two-dimensional domain $D: r<R(\theta)$. An inequality of the form (1) holds for all harmonic functions $u$ if and only if the identity

$$
\begin{aligned}
& R(\theta)\left(R(\theta)^{2}+R^{\prime}(\theta)^{2}\right)^{1 / 2} \frac{\partial G}{\partial n}(R(\theta), \theta) \\
& \quad=R(\theta+\pi)\left(R(\theta+\pi)^{2}+R^{\prime}(\theta+\pi)^{2}\right)^{1 / 2} \frac{\partial G}{\partial n}(R(\theta+\pi), \theta+\pi)
\end{aligned}
$$

1 This research was supported by NSF Grant No. GP-2280. 
holds for all 0 . If (2) is satisfied, (1) holds with $M=1$.

Proof. Let $a$ be a constant such that

$$
\frac{R(\theta+\pi) u(R(\theta), \theta)+R(\theta) u(R(\theta+\pi), \theta+\pi)}{R(\theta)+R(\theta+\pi)} \leqq a
$$

for all $\theta$.

We write the representation

$$
u(O)=-\oint_{r=R(\theta)} u \frac{\partial G}{\partial n} d s
$$

in the form

$$
u(O)=a-\int_{0}^{2 \pi}[u(R(\theta), \theta)-a] \frac{\partial G}{\partial n}(R(\theta), \theta) \frac{d s}{d \theta} d \theta .
$$

In the identity

$$
\begin{aligned}
& \int_{0}^{2 \pi} f(\theta) g(\theta) d \theta=\frac{1}{2} \int_{0}^{\pi}\{[f(\theta)+f(\theta+\pi)][g(\theta)+g(\theta+\pi)] \\
&+[f(\theta)-f(\theta+\pi)][g(\theta)-g(\theta+\pi)]\} d \theta
\end{aligned}
$$

we let

$$
\begin{aligned}
f(\theta) & =\frac{u(R(\theta), \theta)-a}{R(\theta)}, \\
g(\theta) & =-R(\theta) \frac{\partial G}{\partial n}(R(\theta), \theta) \frac{d s}{d \theta} \\
& =-R(\theta)\left(R(\theta)^{2}+R^{\prime}(\theta)^{2}\right)^{1 / 2} \frac{\partial G}{\partial n}(R(\theta), \theta) .
\end{aligned}
$$

Then $g(\theta) \geqq 0$. By (3),

$$
f(\theta)+f(\theta+\pi) \leqq 0 .
$$

Thus,

$$
u(O) \leqq a+\frac{1}{2} \int_{0}^{\pi}[f(\theta)-f(\theta+\pi)][g(\theta)-g(\theta+\pi)] d \theta .
$$

Equality holds if $f(\theta)+f(\theta+\pi) \equiv 0$; that is, for those boundary values $u(R(\theta), \theta)$ satisfying $R(\theta+\pi) u(R(\theta), \theta)+R(\theta) u(R(\theta+\pi), \theta+\pi)=a[R(\theta)+R(\theta+\pi)]$. 
If $f(\theta)$ is made to satisfy only this condition, the function $f(\theta)-f(\theta+\pi)$ is completely arbitrary for $0 \leqq \theta<\pi$. The right-hand side of (8) and therefore also $u(O)$ can be made arbitrarily large unless $g(\theta)-g(\theta+\pi)$ $=0$. This is the condition (2).

If (2) is satisfied, (8) becomes $u(O) \leqq a$, which is (1) with $M=1$.

We remark that (2) is certainly satisfied if the symmetry condition

$$
R(\theta+\pi)=R(\theta)
$$

holds. This means that the point $O$ bisects each chord through it. This is true at the center of an ellipse, or of a parallelogram. In such a case we find that

$$
u(O) \leqq \max \frac{1}{2}[u(R(\theta), \theta)+u(R(\theta+\pi), \theta+\pi)] .
$$

We can now prove:

THEOREM. If a bound of the form (1) for harmonic functions $u$ holds at each point $O$ of a convex domain $D$ with smooth boundary $C$, then $C$ is a circle.

Proof. We consider the chord $P Q$ connecting any two boundary points $P$ and $Q$. Let its length be $d$, and let $O$ be the point on this chord at distance $\delta$ from $Q$.

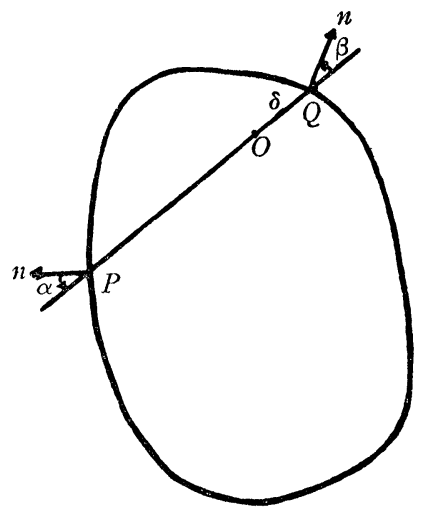

Let the chord make angles $\alpha$ and $\beta$, respectively, with the normals at $P$ and $Q$.

By hypothesis, (1) holds at $O$. Hence by the lemma we have

$$
\frac{(d-\delta)^{2}}{\cos \alpha} \frac{\partial G}{\partial n}(O, P)=\frac{\delta^{2}}{\cos \beta} \frac{\partial G}{\partial n}(O, Q) .
$$

We let $O$ approach $Q$ by making $\delta \rightarrow 0$. It is easily seen that 


$$
\frac{\partial G}{\partial n}(O, Q)=\frac{-1}{\pi \delta} \cos \beta+O(1)
$$

(The leading term comes from Green's function for the half-plane.) On the other hand, since $(\partial G / \partial n)(O, P)=0$ for $O$ on $C$,

$$
\frac{\partial G}{\partial n}(O, P)=-\cos \beta \frac{\partial^{2} G}{\partial n_{P} \partial n_{Q}}(P, Q)+O\left(\delta^{2}\right) .
$$

Dividing (9) by $\delta$ and letting $\delta \rightarrow 0$, we find

$$
d^{2} \frac{\partial^{2} G}{\partial n_{P} \partial n_{Q}}(P, Q) \frac{\cos \beta}{\cos \alpha}=\frac{1}{\pi} .
$$

The function $\partial^{2} G / \partial n_{P} \partial n_{Q}$ is symmetric in $P$ and $Q$. Letting $\delta \rightarrow d$, we find the same equation with $\alpha$ and $\beta$ interchanged. Hence $\cos \alpha$ $=\cos \beta$. This is true for all $P$ and $Q$ on $C$. Letting $Q \rightarrow P$ on $C$ and using the fact that $\beta$ is a continuous function of $Q$, we find that $\alpha=\beta$.

An elementary exercise in differential geometry shows that $\alpha=\beta$ for all $P$ and $Q$ on $C$ implies that $C$ is a circle. This proves the theorem.

REMARK. If we restrict our attention to non-negative $u$ :

$$
u(R(\theta), \theta) \geqq 0,
$$

the inequality (8) does lead to a bound of the form (1) with the best possible constant

$$
\begin{aligned}
M=1+\int_{0}^{\pi} \max \left\{\frac{1}{R(\theta+\pi)}[g(\theta)-g(\theta+\pi)]\right. & \\
& \left.\frac{1}{R(\theta)}[g(\theta+\pi)-g(\theta)]\right\} d \theta .
\end{aligned}
$$

However, the evaluation of this constant requires rather detailed information about the kernel $\partial G / \partial n$, which is difficult to come by.

In this case the maximum principle gives (1) with

$$
M=1+\max _{0 \leqq \theta \leqq 2 \pi}\left\{\frac{R(\theta+\pi)}{R(\theta)}\right\},
$$

which is just what one obtains by means of crude estimates for the Green's function in (10).

The analogous results in $n$ dimensions can be proved in the same manner. 


\section{BIBLIOGRAPHY}

1. J. Barta, Sulla risoluzione del problema di Dirichlet per il cerchio o per la sfera, Atti Acad. Naz. Lincei Mem. (6) 6 (1937), 783-793.

2. - Bornes pour la solution du problème de Dirichlet, Bull. Soc. Roy. Sci. Liège 31 (1962), 15-21.

3. - Sur une certaine formule qui exprime des bornes pour la solution du problème de Dirichlet, Bull. Soc. Roy. Sci. Liège 31 (1962), 760-766.

4. W. H. Malmheden, Eine neue Lösung des Dirichletschen Problems für sphärische Bereiche, Kung1. Fysiogr. Sällsk. i Lund Förh. 4 (1934), no. 17, 1-5. 111.

5. R. J. Duffin, A note on Poisson's integral, Quart. Appl. Math. 15 (1957), 109-

UNIVERSity OF MinNEsota

\section{A NOTE ON THE FUNDAMENTAL THEORY OF ORDINARY DIFFERENTIAL EQUATIONS}

BY GEORGE R. SELL ${ }^{1}$

Communicated by H. Antosiewicz, February 19, 1964

In this note we present some results on various problems connected with ordinary differential equations which do not necessarily satisfy a uniqueness condition. Using the concept of an integral funnel we are able to generalize the classical theorem on continuity with respect to initial conditions. This then leads to a reformulation of the problem of classifying the solutions of a given differential equation. That is, it is shown that every continuous vector field $f(x)$ on $W$ gives rise to a bicontinuous injection of $W$ into a space of functions $H$, and consequently the problem of classifying solutions is equivalent to the problem of characterizing this family of bicontinuous injections. A detailed discussion, with proofs, will appear later.

1. Introduction. Let us consider the differential equation

$$
x^{\prime}=f(x)
$$

where $f$ is defined and continuous on some open, connected set $W$ in $R^{n}$, real $n$-space. We shall let $W^{*}=W \cup\{\omega\}$ denote the one-point compactification of $W$. There is then at least one solution $\phi(p, t)$ of (1) through every point $p \in W$ with $\phi(p, 0)=p$. Moreover, every solution is defined on some maximal interval $J_{p}$ where either $J_{p}=R^{1}$ or $\phi(p, t) \rightarrow\{\omega\}$ as $t \rightarrow b d y J_{p}$. It should be noted that since the solutions of (1) may not be unique, the interval $J_{p}$ depends not only on $p$

\footnotetext{
1 The research on this paper was supported in part by a grant from the U. S. Army Research Office (Durham).
} 